\title{
Analysis and Design of GRB System Based on WSRF Web Service
}

\author{
Ying Zheng \\ Editorial Department of Journal, Inner Mongolia University for Nationalities \\ Tongliao 028043, China \\ Tel: 86-139-4759-5286 E-mail: 13947595286@163.com
}

\author{
Received: December 30, $2011 \quad$ Accepted: January 16, $2012 \quad$ Published: March 1, 2012 \\ doi:10.5539/cis.v5n2p94 URL: http://dx.doi.org/10.5539/cis.v5n2p94
}

\begin{abstract}
One advantage of WSRF Web Service is to establish loosely connective system by itself. This kind of system is easier to be updated than strongly connective system, and its demand of the system is little in real implementation of Web Service. Loose connection is a favorite design pattern for a researcher in software design. GRB (Grid Resource Broker) system has to consider how the control flow flows from a service to another one and emphasize on designing the implementation of indexing service in GT5(Globus toolkits 5). GRB system centers on resource administration in computing grid environment and carries out the research surrounding numerous key technologies. The resource publication, querying resource, spending resource, file transfer service and system security problem of GRB system are analyzed and designed, and thus some exploration on the research related to grid computing and its grid application is done.
\end{abstract}

Keywords: Grid Resource Broker, OGSA, WSRF Web Service

\section{Introduction}

Grid computing, as a kind of infrastructure with rapid development, can share large-scale distributed computing resource, storage resource, data resource, software resource, device resource, human resource and so on. Grid computing can break through current limitation of computing mode and realize large-scale collaborative scientific computing and collaborative problems solving, and it becomes a new distributed computing mode, which is recognized as the third information technology wave after internet and Web, and is one of the important fields of research and application of next generation's internet technology. Nowadays, grid computing is concerned by many researchers and large enterprises at home and abroad, and attracts huge capital investment, which has become a hot spot research and advanced problem.

In 2000, Once Web Services standard were proposed, because of its wide Web industrial base, it was immediately concerned by the project Globus which was the most successful about grid research in scientific research field, and was combined to OGSA (Open Grid Service Architecture) standard, and then was evolved and reconfigured to grid resource frame-WSRF(Web Services Resource Frame) gradually (http://www.oasis-open.org/, 2010), which was proposed in the beginning of 2004. Currently the research work based on this frame is being carried out globally in full swing (WANG Huifang, 2007).

The characteristics of grid such as large-scale, heterogeneous, dynamic, distributed and autonomous make its resource administration complex and difficult to implement with high efficiency. Meanwhile, the urgent need of usability and accessibility make the research on resource administration of grid computing more crucial and necessary.

The article centers on resource administration in computing grid environment and carries out the research surrounding numerous key technologies, and thus some exploration on the research related to grid computing and its grid application is done.

\section{The Developmental Trend of Grid Technology}

According to the whole development of grid technology, its approximate trend is (HUANG Changqin, 2005) (YIN Feng, 2006):

1) Standardization: In January 2004, the combination of Web Services standard and OGSA standard (http://www.ggf.org/, 2011) - WSRF was proposed and it will become the new standard of network in future. 
2) Technology Convergence: The project group of Globus saw huge potential of Web Services and promptly turned the development of Globus Toolkit (http://www.globus.org/, 2011) to Web Services platform (GONG Qiang, 2008), and attempted to unify the others in network world through OGSA. On the basis of OGSA, all external functions of grid are embodied by grid services and realize the functions including the description, search, access and information transfer of those services with the help of some existing technology irrelevant with platform such as XML, SOAP, WSDL, UDDI and so on. Then the heterogeneity of all the platforms and their technology used are blocked. While users have access to grid service, they do not need to pay attention whether the service is provided by CORBA or .Net (LI Wei, 2007).

3) Large-scale: American government made a huge investment in implementing the grid program applied in entire American various fields. In August 2001, IBM announced they would spend 4 billion USD on Gird Computing Initiative to prompt their global grid strategy and became a chief partner of Globus, positively participating in the formulation of grid computing standard such as OGSA standard. China also spent billions Yuan on developing ChinaGrid, which can be seen the trend of nationalization and globalization of grid development.

\section{The Overview of Web Services}

Web Services, is another distributed computing technology, similar to CORBA, RMI and EJB, through which client/server application can be established. But compared to CORBA, RMI, EJB and many other technologies, Web Service has following advancement:

1) Web Services is based on standard XML language, so it has the independence of platform and language, which means that client program can be developed by $\mathrm{C}++$ under Windows, but Web Service can be developed by Java under Linux.

2) Most of Web Service adopts HTTP to deliver information (for example, service request and response). The largest advantage of this way is establishing the application of an entire internet.

3) One advantage of Web Service is to establish loosely connective system by itself. This kind of system is easier to be updated than strongly connective system, and its demand of the system is little in real implementation of Web Service. Loose connection is a favorite design pattern for a researcher in software design. Because Web Service is driven by information and delivers information and confirms the port based on natural XML syntax, it is an ideal choice of establishing loose system.

Of course, Web Service also has some shortages:

1) High cost. All data transmission is based on XML and it is obvious that the efficiency is lower than directly using binary transfers. In other words, Portability is obtained at the cost of efficiency. Even so, the cost is usually accepted by most applications, because the requirement of the system which adopts Web Service for real-time is not high generally.

2) Premature. Web Service is a relatively new technology. Although the core norms about basic language and protocol have been stable, after all, Web Service is an object in a high-speed development. Many standards are still very new, even still under formulation.

Therefore, the design of grid resource broke system based on Web Service and its effective implementation has important value of research currently.

\section{GRB System Analysis and Design}

\subsection{The Main Constitute of GRB System}

1) Resource register: usable resource is registered by agent.

2) Indexing service of resource agent: the indexing service of resource agent collects and settles related information of usable resource. Agent is able to track resource, ensuring the correctness of the information about usable resource.

3) Resource request: The consumer of resource communicates with certain agent server and requests the resource that accords with several parameters.

4) Return resource set: Agent can search a kind of resource that accords with the standard of request through indexing service. There is a gateway before indexing service, which is responsible for explaining requests and performing organizational resource access strategy, where a great deal of detailed information involved by suitable resource can be hidden to seek and positioned.

5) Privacy and integrity: In the scheme, only the privacy and integrity of the communication are considered and the technology of digital signature is adopted. 
6) Audit: It decides whether it is necessary to keep the logs that record interactivities between crucial components, and the information of logs can help recognize and track certain problems and solve some disputes.

\subsection{The Problems to Be Solved}

1) Authentication: in grid computing environment, security is considered firstly. Secure communication is usually authenticated mutually and checks the identity of the client who uses GRB system. As for the method of identity authentication, performance is an important reference standard in application system, because many potential files have to be transferred in the application. Therefore, there are both advantages and disadvantages in the scheme of PKI (Public Key Infrastructure) (C. Adams, S. Farrell, 1999) (http://www. Ietf .org /rfc/rfc3280.txt, 2002) and IBE (Identify based Encryption).

2) The service in container: analyze, accept or reject the service that should be arranged in each container.

3) Resource security strategy: make analysis and judgment on using resource level-oriented security strategy and service level-oriented security strategy.

4) File transfer service: in Globus, single indexing service can be performed, or reliable file transfer (RFT) service can be used. The solution to file transfer is decided by test.

5) Service integration: Which service should be confirmed needs to be written by yourself as well as which service can be replaced by current service. Globus Toolkits contain many services like that and integrate them into your own service through Java WS Core.

6) Authority: decide whether users need to have specified access or WS-Resource through authority. External authentication service can be used, for example, SAML authority. And CAS authority mechanism (Park, Sang M., Chung, Soon M, 2008) provided by Globus also can be used. System performances should be considered comprehensively to decide authority strategy which is used (K. Keahey, V. Welch, 2002) (K. Keahey, V. Weleh, S. Lang, B. Liu, \& S. Meder, 2003).

\subsection{Design Scheme}

Consider how the control flow flows from a service to another one and emphasize on designing the implementation of indexing service in GT5.

1) Resource publication: Firstly consider that designing client application program should be brief and clear. The emphasis of design and implementation is mainly embodied by service. In the machine of resource provider, it is necessary to register local index in agent virtual organization index. If operating GRB system in one machine, two containers need to be operated at the same time: one is agent, the other one is resource provider index.

2) Querying resource: Consider that a client program in the machine of resource provider and two services arranged in agent machine: One service---resource agent service needs to be implemented by program; the other agent index service is undertaken by query index service.

(1) Resource agent service: Responsible for searching certain file with specific name according to the request of price and need file name and its highest price. The solution to implement Web Service resource industrial mode is to put all codes in an independent container, making codes more concise.

(2) Querying indexing service: Consider sending query operation to indexing service.

3) Spending resource: Resource consumer will make use of the searched resource involved by resource provider, resource agent and resource consumer. The implementation from a service to another one should be paid attention as well as the implementation of the flow of events in sequent graph.

4) File transfer service: File transfer service is a service without state, which sends a file to designated consumer. In order to improve the speed of transferring, the file needs to be read into an array of bytes while transferring binary data.

5) The consideration of security: In grid computing environment, security is considered firstly. Because the collaboration based on grid involves the people and resource in various management domains, they share important resource in grid between each other. In turn, the consideration of security in operation can influence the performance of system. While optimizing performance, how to reduce the possibility of misoperation of system to the minimum is a difficulty in a system design.

For grid computing, grid identity authentication is one of the core problems of grid application (http://www.lobus.org/toolkit/docs/4.0/security/delegation, 2010) (http://www.schneier.com/book-practical.html). Because a grid usually crosses several virtual organizations and resource can be visited by different organizations, it will inevitably be faced with various challenges to the aspect of security: it must be ensured that only users 
permitted can have access to resource and the members' identities in the grid must be authenticated, which means to ensure that every user in grid application can be identified appropriately, and ensure the integrality and privacy of the grid communication data; users may relegate their own limits of authority to others and carry out agent operation.

\section{Conclusions}

One advantage of Web Service is to establish loosely connective system by itself. This kind of system is easier to be updated than strongly connective system.

GRB system has to consider how the control flow flows from a service to another one and emphasize on designing the implementation of indexing service in GT5.

GRB system centers on resource management in computing grid environment and carries out the research surrounding numerous key technologies. The resource publication, querying resource, spending resource, file transfer service and system security problem of GRB system are analyzed and designed, and thus some exploration on the research related to grid computing and its grid application is done.

\section{References}

Bruce chneier. (2003). Practical Cryptography. John Wiley \& Sons. [Online] Available at: http://www.schneier.com/book- practical.html

C. Adams, \& S. Farrell. (1999). Internet X.509 Publickey Infrastructure. IETF RFC 2510. [Online] Available at: www.ietf.org/rfc/rfc2510.txt?number=2510.

Global Grid Forum. (GGF). (2011). [Online] Available at: http://www.ggf.org/

GONG Qiang. (2008). Grid Research current situation feature and development trend (continued). Information Technology, 6, 1-7. (in Chinese)

GT4.0. Security: Delegation Service. (2010). [Online] Available at: http://www.lobus.org/toolkit/docs/4.0/security/delegation.

HUANG Changqin. (2005). A Study on Several Issues of Task Management in Computational Grids. Ph.D. thesis, Zhejiang University, 2005.3 (in Chinese)

Internet X. 509 Public key Infrastructure. (2002). Certificate and Certificate Revocation List (CRL) Profile. [Online] Available at: http://www.Ietf.org/rfc/rfc3280.txt

K. Keahey, \& V. Welch. (2002). Fine-Grain Authorization for Resouree Management in The Grid Environment. In Proceedings, Grid Computing 2002 (GRID2002), Third International Workshop,Lecture Notes in Computer Seienee: Security and Policy Management, 2536, Springer-Verlag, Baltimore, MD, U.S.A., 2002-2011.

K. Keahey, V. Weleh, S. Lang, B. Liu, \& S. Meder. (2003). Fine- Grain Authorization Policies in the GRID: Design and Implementation. The $1^{\text {st }}$ International Workshop On Middleware for Grid Computing, 170-177.

LI Wei. (2007). Research of Grid Scheduling Model Based on Cooperative Game. Master's thesis, Jilin University, 2007.04 (in Chinese)

OASIS. (2010). http://www.oasis-open.org/

Park, Sang M., Chung, \& Soon M. (2008). Enhanced CAS certificate for metadata-based access control in grids. Proceedings - International Conference on Tools with Artificial Intelligence, ICTAI, v 2, p 323-329, 2008, Proceedings - 20th IEEE International Conference on Tools with Artificial Intelligence, ICTAI'08.

The Globus Alliance. (2011). http://www.globus.org/

WANG Huifang. (2007). Research on Grid-Based Information Integration System. Master's thesis, Shanghai Jiaotong University, 2007.1 (in Chinese)

YIN Feng. (2006). Campus Grid Technology Research Satisfying QoS. Sichuan University doctoral thesis, 2006.9 (in Chinese) 